\title{
Section on Urology - American Academy of Pediatrics San Francisco 10.-12. Oktober 1992
}

\author{
J. Fichtner \\ Department of Urology, Stanford University, USA
}

\section{Hypospadiekorrektur und plastisch-rekonstruktive Urologie}

Sowohl Duckett (Philadelphia) als auch Ransley stellten ihre sehr positiven Ergebnisse mit der Verwendung von Mundschleimhaut zur Harnröhrenrekonstruktion vor.

Ransley berichtete über 22 Kinder (davon 19 Hypospadiekrüppel), die während des letzten Jahres mit Mundschleimhaut versorgt wurden, davon 16 mit Einschluß des Meatus, wobei in keinem Fall eine Meatusstenose beobachtet wurde, 3 Patienten entwickelten postoperativ eine Fistel. Bei geplanter Bildung eines Rohres entnimmt er die Mundschleimhaut aus der Wange, bei Bildung eines Onlay-Patches von der Unterlippe, die Entnahme eines zusammenhängenden Streifens von der einen Wange über die Unterlippe zur gegenüberliegenden Wange ergibt ein komplettes Harnröhrenrohr von ca. $12 \mathrm{~cm}$ Länge.

Duckett stellte vergleichende histopathologische Untersuchungen von Mundschleimhaut, Vorhaut und Blasenmukosa vor.

Die Mundschleimhaut besitzt bei weitem das dickste Epithel (wichtig für strukturelle Integrität) und gleichzeitig die dünnste Lamina Propria/Dermis (wichtig für schnelle Revaskularisierung).

Blasenmukosa und Vorhaut hingegen weisen ein genau entgegengesetztes Muster auf, was im Hinblick auf die Blutversorgung und Stabilität eher ungünstig erscheint.

Ransley stellte weiterhin seine Serie von 92 Patienten vor, die mit einem Blasenschleimhautgraft versorgt wurden.

Hier zeigte sich zwar in insgesamt $91 \%$ der Patienten schlußendlich ein zufriedenstellendes Ergebnis, welches allerdings erst nach mehrfachen Korrekturen der häufigen Komplikationen erzielt wurde. $61 \%$ der Patienten benötigen zwischen 1 und 8 Folgeoperationen (Durchschnitt 2,8) zur Korrektur von Fisteln, Meatusstenosen und meatalen Mukosaprolapsen. Ransley unterstrich in der Diskussion, daß er selbst vor Analyse der Da-

Akt. Urol. 24 (1993) 163-164

(c) Georg Thieme Verlag Stuttgart · New York ten mit einer fast $100 \%$ igen Komplikationsrate gerechnet habe, für ihn sei jetzt eindeutig die Mundschleimhaut die Methode der Wahl.

\section{Blasenexstrophie}

Mehrere Vorträge beschäftigten sich mit der Frage der Harnkontinenz nach Blasenrekonstruktion.

Jeffs (Baltimore) stellt die Serie aus dem Johns Hopkins von 72 Patienten vor, bei denen nach initialem Blasenverschluß und späterem „Young-Dees“ keine zusätzliche Blasenaugmentation vorgenommen wurde. Die Analyse der Kontinenz zeigt eine eindeutige Korrelation mit dem Alter: insgesamt $92 \%$ der Kinder sind sozial kontinent $=$ ca. 3 Stunden, nur $20 \%$ der 5-10jährigen sind day and night trocken, verglichen mit einer day and

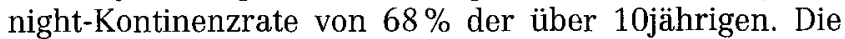
zunehmende Harnröhrenlänge, die wachsende Prostata mit zunehmendem Alter und der Lernprozeß können als Gründe dafür aufgeführt werden.

\section{Vesiko-uretero-renaler Reflux}

Die endoskopische Behandlung des Refluxes impliziert mit den heute zur Verfügung stehenden Substanzen zur submukösen Injektion nach wie vor Risiken was die Migration der Partikel und Langzeitergebnisse angeht.

Aaronson u. Mitarb. (Chareston) berichteten über eine experimentelle Studie an Hunden, bei denen zahlreiche Teflon-Nester im Gehirn 2 Jahre nach submuköser Tefloninjektion festgestellt wurden. Zusätzlich zeigten sich in angrenzenden Hirnarealen Demyelinisierungen mit interstitiellen Neuromucinansammlungen, was offensichtlich eine Folge von Embolisierungen der Hirngefäße durch Teflonpartikel mit folgender Störung der Mikrozirkulation ist.

Verantwortlich für die Migration der Partikel ist die Teilchengröße, Aaronson betonte, daß sowohl Kollagen als auch Siloxan deshalb wahrscheinlich dem gleichen Migrationsprozeß unterliegen und deshalb nicht klinisch eingesetzt werden sollten.

Keine der heute zur Verfügung stehenden Substanzen erfüllt alle theoretisch nötigen Anforderungen: einfache Applikation, Erhalt des Volumens, keine Migration und keine antigenen Eigenschaften. 
Atala (Boston) unterstrich die Möglichkeit der laparoskopischen Refluxchirurgie im Tierexperiment (Minipig) mit einer modifizierten Technik nach „Lich-Gregoir". In der Diskussion zeigte sich eindeutig, daß die extravesikale Refluxchirurgie (Lich-Gregoir, ggf. mit Advancement) gerade auch wegen der leichten Úbertragbarkeit auf die laparoskopische Operation gegenwärtig eine Renaissance in den Vereinigten Staaten erlebt. Schulman (Brüssel) wies auf seine Serie von über 1000 Gregoir-Operationen hin.

\section{Subpelvine Stenose}

Die Schwierigkeiten der Beurteilung der klinischen Signifikanz einer kindlichen Harntransportstörung mit den heute zur Verfügung stehenden diagnostischen und bildgebenden Verfahren stellt ein zentrales Problem bei der Entscheidung für oder gegen einen operativen Eingriff dar.

Ransley u. Mitarb. (London) stellten eine prospektiv randomisierte Studie an 65 Kindern mit unilateraler partieller Obstruktion vor (Pelvis $>15 \mathrm{~mm}$, dilatierte Kelche, Funktion über $40 \%$ in der seitengetrennten Clearance). Von den 34 zur Nierenbeckenplastik randomisierten Kindern zeigten 32 eine stabile Nierenfunktion, 2 wiesen eine leichte Verschlechterung bei verbesserter Morphologie des Hohlsystems auf. 31 Kinder wurden konservativ behandelt, bei 6 Kindern (Pelvisdiameter bei allen größer $20 \mathrm{~mm}$ ) mußte aufgrund abnehmender Funktion eine Operation vorgenommen werden. Die Analyse der diagnostischen Parameter (Clearance, Sonogramm, IVP, Whitaker-Test) ergab kein eindeutiges Kriterium, um die Kinder zu identifizieren, die eine Operation benötigen. Bei 4 von den 6 Kindern, die aus der konservativen Gruppe eine Nierenbeckenplastik benötigten, zeigte beispielsweise der Whitaker-Test niedrige Druckwerte.

\section{Augmentation}

Verschiedene Gruppen stellten ihre Erfahrungen mit der Gastrozystoplastik vor. Mitchell (Seattle) wies auf das Risiko des Dysurie- und Hämaturie-Syndroms (DHS) hin, das er in ca. $17 \%$ seiner bisher $57 \mathrm{~Pa}-$ tienten mit Gastrozystoplastik beobachtet hat und nur partiell medikamentös kontrollieren konnte. Wenngleich theoretisch die aktive Sekretion von Magensäure gerade bei niereninsuffizienten Patienten von Vorteil ist, um eine Azidose zu vermeiden, kann es hingegen auf der anderen Seite zu schweren hypochlorämischen, hypokaliämischen metabolischen Alkalosen kommen.

Zwei Präsentationen wiesen auf die teilweise lebensbedrohlichen intraoperativen Latexallergien bei mehrfach voroperierten Patienten (besonders Spina bifida Patienten) hin und empfehlen die routinemäßige präoperative Durchführung eines radioallergosorbent test (RAST) in gefährdeten Patientengruppen.

\section{Kryptorchismus}

Hadziselimovic untersuchte in einer randomisierten Studie den Effekt von HCG und Buserelin auf die Sertolizellen, und wies eine Abnahme der Sertolizellen bei alleiniger HCG-Behandlung nach, die durch die zusätzliche Verabreichung von Buserelin aufgehoben wurde.

\section{Urodynamik}

Ambulante Urodynamik mit physiologischer Blasenauffüllung, weniger invasiver Untersuchung und sensitiverer Technik wurde von der Gruppe aus der Great Ormond Street favorisiert.

Bloom und McGuire (Ann Arbor) stellten den Stress Leak Point Pressure (LPP-S) zur Beurteilung der Kompetenz des Blasenhalses und der proximalen Harnröhre vor. Im Gegensatz zum Leak Point Pressure, der ein passiver Test der outlet-resistance und Parameter für das Risiko einer Schädigung des oberen Harntraktes ist, kann der LPP-S als provokativer Test (Valsalva) Information bieten bei Kindern, bei denen eine Inkompetenz des Blasenhalses bei Anstieg des intra-abdominalen Druckes vorliegt. Gerade myelodysplastische Patienten weisen oft paradoxerweise einen erhöhten LPP auf bei gleichzeitig vorliegender schwerer Streßinkontinenz.

\section{Grundlagenforschung}

Baskin und McAninch (Philadelphia, San Francisco) versuchten, in einem interessanten Versuchsaufbau in Zellkulturen die Genese von Harnröhrenstrikturen nachzuvollziehen.

Durch ein initiales Trauma kommt es zu einer Disruption der Fibroblasten, welche zu einer andauernden Stimulierung der Kollagensynthese (Typ I und III) mit folgender Narbenbildung durch diese Zellen führt. Dies könnte auch erklären, warum Strikturen teilweise erst Jahre nach dem initialen Trauma klinisch manifest werden.

Cowan und Shortliffe (Stanford) zeigten, daß eine Harnwegsinfektion zu erhöhten pelvinen Druckwerten führt, die durch die Gabe von Oxybutinin gesenkt werden können.

\section{Dr. J. Fichtner}

Department of Urology

Stanford University

USA 